\title{
Perceived target displacement as a function of field movement and asymmetry
}

\author{
ROBERT C. SUGARMAN ${ }^{2}$ AND WALTER COHEN \\ STATE UNIVERSITY OF NEW YORK AT BUFFALO
}

In two experiments induced movement of an object was produced to demonstrate that movement of the background influences the perceived localization of the object in space. The Roelofs (asymmetry) effect could be used to explain only part of the shift in localization in Experiment 1. The asymmetry effect was excluded from Experiment 2 by the procedure employed. It was concluded that the Roelofs effect is a sufficient, but not necessary, condition for the effects of induced movement to occur, and that relative displacement of the target and background plays an important part in the illusion. Furthermore, it was shown that the effects of induced movement can occur even when the border of the background remains stationary.

Evidence has recently been presented by Brosgole $(1966 \mathrm{a}, 1966 \mathrm{~b}, 1967)$ to support the hypothesis that induced movement is the result of a change in the subjective median plane rather than the result of a displacement of the object relative to the background. The subjective median plane shifts towards the center of the visual array when the center does not coincide with the true median plane (Roelofs effect). Such findings have been reported by Roelofs (1935), and also by Wapner and Werner (1957) and Loemker (reported by Carr, 1935).

This paper will present the results of two studies which do not deny the importance of the Roelois effect in induced movement, but do show that this effect cannot account for all of the subjestive change of the object's position in space which accompanies induced movement. Whereas the task used by Brosgole (1966a) consisted of an adjustment of the object, the task used in the experiments to be reported here required $S$ to point his unseen hand in the direction of the object. Because of its function, the object will be referred to as the target.

\section{Apparatus}

The background was curved so that every point on it was equidistant ( $4 \mathrm{ft}$ ) from S's head. The overall dimensions of the background were approximately $45 \mathrm{deg}$ of visual angle wide by $30 \mathrm{deg}$ high. It was mounted on a curved track so that it could be moved horizontally for a distance of $45 \mathrm{deg}$ at a speed of $2.5 \mathrm{deg} / \mathrm{sec}$. The background stimuli were 48 illuminated $3 / 4$ in. circular spots. These spots could be arranged either as six regularly spaced columns of eight spots each, or in a pseudo-random arrangement in which there was no apparent pattern.

A horizontal strip was missing from the center of the background so that a target (a luminous, $3 / 4$ in. $x 2$ in. rectangle) could be placed within the background. The target also maintained a distance of $4 \mathrm{ft}$ from $S^{\prime}$ s head and could be made stationary so that the target remained in a fixed location with respect to the room, while the background was in motion.

The brightness of the target was $.02 \mathrm{ft}-\mathrm{L}$. The brightness of the spots making up the background ranged between .03 and $.06 \mathrm{ft}-\mathrm{L}$. At these low intensities no reflections could be seen and no afterimages were observed.

$S$ indicated his judgment of the target's position by gripping a pistol-shaped pointer with his right hand, extending his forefinger along the side of the "barrel," and rotating the pointer until it lined up in the same horizontal direction as the target. The pointer was attached to one end of an $11.5 \mathrm{in}$. arm, with the other end serving as the point of rotation. This pivot point was at the center of rotation of the background. The pointer and all but the few inches of the arm around the pivot point were always hidden from S's view. The position of the pointer was displayed on a remotely controlled compass indicator.

S's head was held in place by a biteboard so that his eyes were at the same height as the target and directly above the pointer's center of rotation. The pointer was set at a convenient height to allow free arm movements. Since this height was below eye level, s tried to aim the pointer at a point directly below the target, rather than directly at the target. Between trials a curtain could be closed in front of S to prevent him from seeing the stimuli. In order to insure that $S$ did not get any auditory cues as the background changed position, a masking noise was employed. $S$ was instructed to avoid turning his body or feet away from the straight ahead position. The experiment sessions lasted $50 \mathrm{~min}$. A more complete description of the apparatus may be found in Sugarman (1967).

\section{Subjects}

Ss were both males and females taking part in the experiments in order to fulfill a requirement for their introductory psychology course. All Ss were right-handed and had normal vision without correction (contact lenses were not allowable). Ss were naive with respect to the nature of the experiments.

\section{EXPERIMENT 1}

The target was held in position at $-15,-5,0,5$, or 15 $\mathrm{deg}$, where the $0 \mathrm{deg}$ position is straight ahead of $\mathrm{S}$, the negative distances are to the left of straight ahead, and the positive distances are to the right. The movement of the background was from left to right or from right to 
left. Because of physical limitations of the apparatus the distance traveled by the background could not be as large when the target was at -15 and 15 deg as it could when the target was straight ahead. Therefore, when the target was straight ahead the background was moved 7.5, $15,22.5$, or $30 \mathrm{deg}$ in order to exploit the full range of possible displacements. For the other target positions, background displacements of 7.5 and $22.5 \mathrm{deg}$ were used. The initial and final positions of the background were adjusted so that the target remained as far from the edges of the background as possible, or in other words, as near to the center of the background as possible. So at the beginning of the trial the background was setat a position which depended on the targetposition and on the distance that the background was to travel.

The trial started by turning off the room lights and opening the curtain which had been obstructing $S$ 's view. At this time $S$ grasped the pointer, which was at its starting position about $70 \mathrm{deg}$ to the right of straight ahead, and bit the biteboard. The target and background were then turned on simultaneously without movement of the background. The stationary background and target remained on for a duration equal to the time required to move the background later in the trial. For example, if the displacement of the background was to be $15 \mathrm{deg}$ for that trial, then at $2.5 \mathrm{deg} / \mathrm{sec}$ that movement would require $6 \mathrm{sec}$. The exposure to the stationary stimuli would therefore be set at $6 \mathrm{sec}$. During this time $S$ fixated the target.

After the target and background turned off, $\mathrm{S}$ maintained his direction of gaze and moved the pointer so as to line up with where he was looking (which should be the place where the target was located). When S was satisfied with his judgment, he sounded a buzzer. The experimenter then "froze" the pointer position on the compass indicator by turning off the power to the indicator. After this, $\mathrm{S}$ was signaled to return the pointer to the starting position. Within a few seconds the background and target were again turned on, but this time the background immediately began to move, traveling a distance corresponding to the exposure duration in the first part of the trial. In order to avoid movement aftereffects, the target and background turned off at the same instant that the background stopped moving. $S$ again pointed to the position of the target after it turned off. When signaled that he was satisfied with his judgment, he removed his hand from the pointer (without returning it to the starting position), the curtain was closed, and the room lights were turned on. The $\mathrm{E}$ then recorded the "first pointing" (pre-movement) from the compass indicator, and by reactivating the indicator, he could obtain the position of the "second pointing" (post-movement). Next, S was signaled to return the pointer to the starting position, after which he could rest for the 20 to $30 \mathrm{sec}$ required to set up the next trial.

Each of 20 Ss received all 24 treatment combinations: 12 combinations of target position and background displacement and two directions of movement. A different randomized sequence of trials was used for each S. Thinking that the regularity of the background might influence the strength of the illusion, Ss were divided into two groups. Six males and four females viewed the irregular background, while the remaining five males and five females saw the regular background.

\section{Results}

A preliminary examination of the data indicated that the asymmetry of the background made a difference (the Roelofs effect) in the judgment of the target position after the exposure to the stationary stimuli (first pointings). In particular, it was noted that for the same target position, $S$ pointed more towards the right when the center of the background was to the left of the target, and more towards the left when the center of the background was to the right of the target. This effect occurred even after as short an exposure as $3 \mathrm{sec}$.

In order to eliminate the exaggeration imposed by the asymmetry effect, the analysis was made in the following manner. For each target position and background displacement there was one trial with the movement to the right and one with movement to the left. The background starting position in one trial was the ending position for the other. Let AR represent the asymmetry effect when the background is displaced to the right, and $A L$ when displaced to the left. $M$ will be the perceived displacement due to induced movement and will be assumed to be the same regardless of the direction of movement. If $\mathrm{X}$ is the actual target position, then $\mathrm{S}$ will point to the positions shown in Table 1 (AR, AL, and $M$ are positive numbers).

If one subtracts the first pointing of Trial R-L from that of Trial $L-R$, the remainder is $A L+A R$. Similarly, subtracting the second pointings in the appropriate order gives us $2 M+A L+A R$. The difference between these two quantities is $2 \mathrm{M}$, or twice the perceived displacement due to the illusory movement of the target. It should be pointed out that AL and AR cannot be considered equal, because the initial and final background positions were not, as a rule, equally asymmetric.

Two analyses of variance (ANOV) were carried out. The first examined the effects of background arrangement, target position, background displacement (excluding the 15 and $30 \mathrm{deg}$ displacements for the straight ahead target positions), and type of perceived target dis-

Table 1. Perceived Location of the Target

\begin{tabular}{lcc} 
Direction of Background & $\begin{array}{c}\text { First Pointing } \\
\text { (Pre-Movement) }\end{array}$ & $\begin{array}{c}\text { Second Pointing } \\
\text { (Post-Movement) }\end{array}$ \\
\hline Left to Right (Trial L-R) & $X+A L$ & $X-M-A R$ \\
Right to Left (Trial R-L) & $X-A R$ & $X+M+A L$ \\
Difference & $A L+A R$ & $2 M+A L+A R$ \\
\hline
\end{tabular}

$X=$ Actual target position; $M=$ Perceived displacement attributed to induced movement: $A L=$ Asymmetry effect with the background to the left of the target; $A R=A$ symmetry effect with the background to the right of the target. 


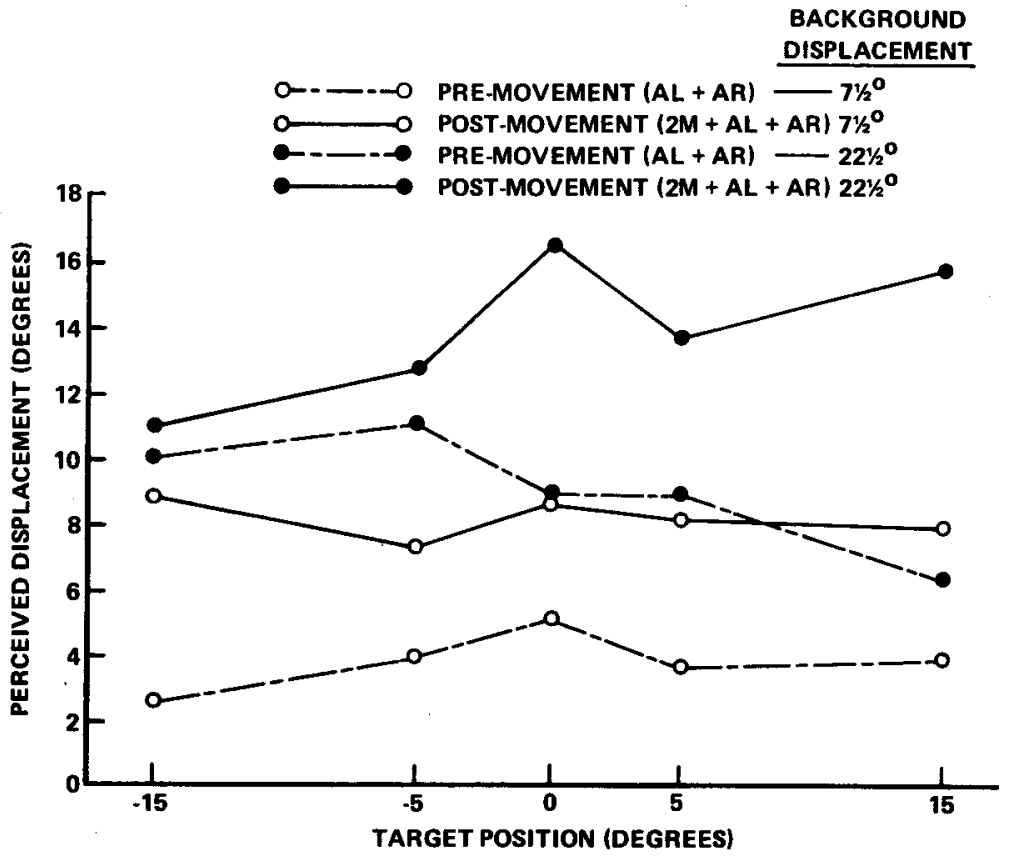

Fig. 1. Perceived target displacement due to the combined effects of induced movement $(M)$ and an asymmetry effect (AL, AR) as compared to that due to the asymmetry effect alone. Each displacement shown is the sum of effects for two related trials. placement (that due only to the Roelofs effect, $A L+A R$, versus the total perceived displacement, $2 M+A L+A R)$. This analysis is presented in Table 2. Figure 1 shows a graph of $A L+A R$ and $2 M+A L+A R$ as a function of background displacement and target position. $M$, averaged over left and right background displacements, may be calculated by taking one half of the difference between the appropriate $A L+A R$ and $2 M+A L+A R$ curves.

Table 2 shows that neither the type of background nor

Table 2. Experiment 1: ANOV Summary for Perceived Displacement (All Target Positions)

\begin{tabular}{|c|c|c|c|}
\hline Source of Variation & $d f$ & MS & $\mathbf{F}$ \\
\hline Background Arrangement (B) & 1 & 510.2 & 1.29 \\
\hline Subjects within B (S) & 18 & 395.7 & \\
\hline Torget Position (T) & 4 & 31.8 & 1.47 \\
\hline$B \times T$ & 4 & 22.0 & 1.01 \\
\hline$T \times S$ & 72 & 21.7 & \\
\hline Amount of Background Displocement $(D)$ & 1 & 2993.0 & 41. $10 * *$ \\
\hline$B \times D$ & 1 & 54.2 & 0.74 \\
\hline$D \times S$ & 18 & 72.8 & \\
\hline$T \times D$ & 4 & 6.3 & 0.28 \\
\hline$B \times T \times D$ & 4 & 7.3 & 0.33 \\
\hline$T \times D \times S$ & 72 & 22.2 & \\
\hline Pre- vs Post-movement (P) & 1 & 2092.0 & $12.32 * *$ \\
\hline$B \times P$ & 1 & 0.5 & 0.00 \\
\hline$P \times S$ & 18 & 169.8 & \\
\hline$T \times P$ & 4 & 51.3 & 0.74 \\
\hline$B \times T \times P$ & 4 & 9.1 & 0.13 \\
\hline$T \times P \times S$ & 72 & 68.8 & \\
\hline$D \times P$ & 1 & 4.0 & 0.08 \\
\hline$B \times D \times P$ & 1 & 52.0 & 1.09 \\
\hline$D \times P \times S$ & 18 & 47.6 & \\
\hline$T \times D \times P$ & 4 & 93.3 & 1.52 \\
\hline$B \times T \times D \times P$ & 4 & 76.0 & 1.24 \\
\hline$T \times D \times P \times S$ & 72 & 61.6 & \\
\hline
\end{tabular}

$* * p<.01$ the position of the target made a difference. The two factors which were significant indicate, in conjunction with Fig. 1, that there is a perceived target displacement due to the movement of the background which is greater than that which can be explained solely in terms of the asymmetry (Roelofs) effect, and that both of these effects increase as the amount of background displacement increases.

The second ANOV included only the straight ahead target position, but examined all four background displacements. This analysis is presented in Table 3 , and shown pictorially in Fig. 2.

The results of the ANOV for the various background displacements at the straight ahead target position (Table 3) are similar to the first analysis, except now the interaction between the background displacement and the type of perceived target displacement effects is significant. As seen in Fig. 2, the total perceived displacement increases almost linearly as the background displacement is increased, while the asymmetry effect is unaffected by increases in background displacement beyond 15 deg (or equivalently, by increases in exposure durations beyond $6 \mathrm{sec}$ ).

To summarize briefly, it was found that during induced movement, Ss not only perceived the stationary target to be in motion, but also perceived the target as changing its location in space. The perceived target displacement may be accounted for only in part by the Roelofs effect. Brosgole did not come to this conclusion because he found no significant difference in the magnitude of the induced movement effect as compared to the Roelofs effect obtained with a stationary background, however his results were in a direction consistent with the data of the present study. 


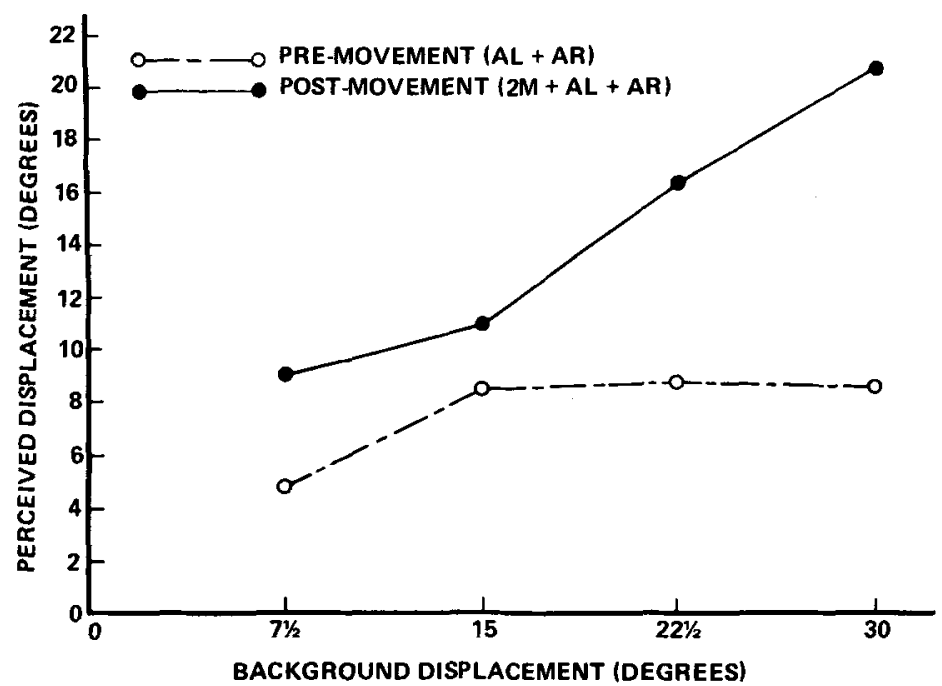

Fig. 2. Perceived displacement of a target (in the straight ahead position) due to the combined effects of induced movement (M) and an asymmetry effect (AL, AR) as compared to that due to the asymmetry effect alone. Each displacement shown is the sum of effects for two related trials.

\section{EXPERIMENT 2}

An alternate means of producing induced movement is exemplified by the illusory movement of the moon as the clouds pass by it. In that case the clouds form a moving background, but the border is fixed since only the clouds in front of the moon are illuminated. A similar situation was created by slightly modifying the apparatus of Experiment 1. Two shields were placed in front of the stimuli, about $2.5 \mathrm{ft}$ from $\mathrm{S}$. The space between the shields was fixed so that only a segment of the background $15 \mathrm{deg}$ wide was visible when viewed binocularly by $\mathrm{S}$. The irregular background was used so that for any position of the background an approximately constant number of spots would be seen in the opening. The target was placed in any of three positions: $-7.5,0$, and $7.5 \mathrm{deg}$. The shields could also be moved so that the target always appeared in the center of the exposed background.

As in Experiment 1, the background was moved from right to left and left to right for each combination of target location and background displacement. The background displacements were $7.5,15,22.5$, and $30 \mathrm{deg}$, corresponding to exposure durations of $3,6,9$, and 12 sec. The procedure was the same as for the "second

Table 3. Experiment 1: ANOV Summary for Perceived Displacement (Target at Straight Ahead Position)

\begin{tabular}{|c|c|c|c|}
\hline Source of Variation & df & MS & $\mathrm{F}$ \\
\hline Background Arrangement (B) & 1 & 140.2 & 0.45 \\
\hline Subjects within B (\$) & 18 & 311.4 & \\
\hline Amount of Background Displacement (D) & 3 & 470.7 & $15.39 * *$ \\
\hline$B \times D$ & 3 & 28.8 & 0.74 \\
\hline$D \times 5$ & 54 & 30.6 & \\
\hline Pre-vs Post-movement (P) & 1 & 1627.0 & $39.30 * *$ \\
\hline $\mathrm{B} \times \mathrm{P}$ & 1 & 74.2 & 2.28 \\
\hline$P \times S$ & 18 & 41.4 & \\
\hline$D \times P$ & 3 & 186.8 & $3.35^{*}$ \\
\hline$B \times D \times P$ & 3 & 99.9 & 1.79 \\
\hline$D \times P \times S$ & 54 & 55.7 & \\
\hline
\end{tabular}

pointings" of Experiment 1, with the pre-movement pointing eliminated since the movement of the background caused no change in the relative position of the target with respect to the background borders.

Each of $11 \mathrm{Ss}$, seven males and four females (not the same as in Experiment 1), were exposed to the $24 \mathrm{com-}$ binations of target position, exposure duration, and direction of movement. A different randomized sequence of trials was used for each $\mathrm{S}$.

\section{Results}

The ANOV for this experiment is presented in Table 4. The significant effects were direction of movement and the interaction between direction and target position. Unlike Experiment 1, the amount of background displacement (exposure duration) did not exert a significant influence on the localization of the target.

Figure 3 shows the mean localization error as a function of target position and direction of travel of the background (the data has been averaged over the four magnitudes of background displacement). Localization error is defined as the judged target position minus the actual target position and is used to simplify the data. From this graph, one finds that the significant F tests represent a shift of the apparent target position in the appropriate direction as a result of induced movement, and more of a shift at the off-center targetpositions than at the straight ahead position. In terms of the previous notation, the upper line in Fig. 3 (Right to Left) and the lower line (Left to Right) represent, respectively, $X+M$ and $\mathrm{X}-\mathrm{M}$. The distance between these lines is therefore equal to $2 \mathrm{M}$, i.e., twice the average perceived displacement produced by movement of the background. The fact that the average localization errors are all shifted in the positive direction (to S's right) is an artifact of the pointer's zero setting which corresponded to the physical straight ahead, rather than to the subjective kinesthetic straight ahead. When questioned after the end of the session, Ss reported that the illusion of target movement 


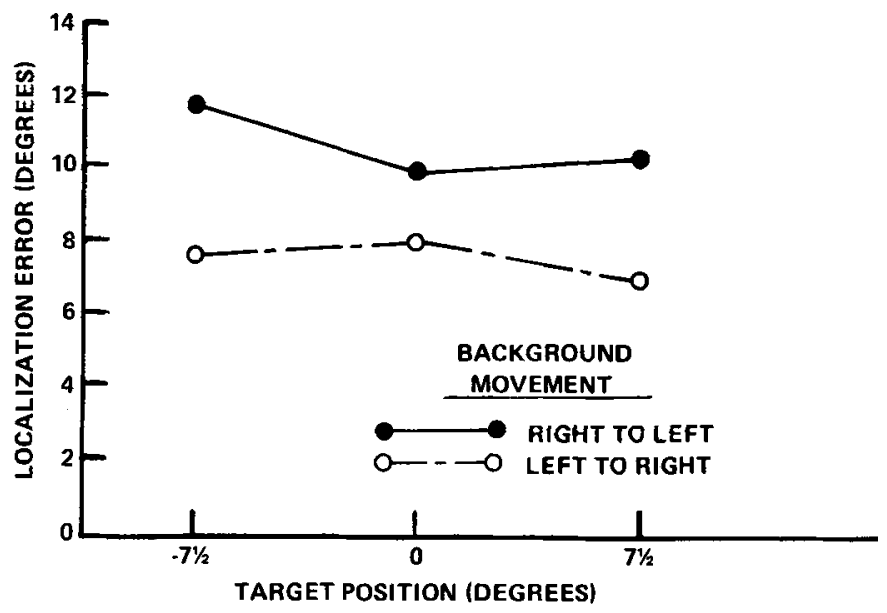

Fig. 3. Error in perceived localization of a target during induced movement when the background border is stationary relative to the target. Localization error equals judged minus actual target position.

was very weak as compared to the reports of Ss in Experiment 1.

\section{CONCLUSIONS}

In both experiments it was shown that movement of a background influenced the perceived localization of the target. The change in localization in neither case may be attributed to the Roelofs effect since this assymmetry effect was factored out in the first experiment and not possible in the second. The contradiction between the results of Experiment 1 and the findings of Brosgole (1966a) are most likely a consequence of the procedural differences.

From the data gathered thus far, it may be concluded that (a) The Roelofs effect is sufficient to produce in-

Table 4. Experiment 2: ANOV Summary for Perceived Displacement

\begin{tabular}{lrrr} 
Source of Voriation & df & \multicolumn{1}{c}{ MS } & $F$ \\
\hline Between Subjects (S) & 10 & 543.91 & \\
Target Position (T) & 2 & 27.31 & 0.94 \\
T X S & 20 & 28.97 & \\
Amount of Background Displacement (B) & 3 & 15.89 & 1.81 \\
B X S & 30 & 8.79 & \\
T X B & 6 & 13.33 & 1.48 \\
T X B X S & 60 & 9.03 & \\
Direction of Backgr ound (D) & 1 & 694.22 & $7.32^{*}$ \\
D X S & 10 & 94.81 & \\
TX D & 2 & 23.09 & $4.32^{*}$ \\
T X X S & 20 & 5.34 & \\
B X D & 3 & 3.29 & 0.29 \\
B X D X S & 30 & 11.20 & \\
T X B X D & 6 & 2.92 & 0.25 \\
T X B X D S & 60 & 11.92 & \\
\hline
\end{tabular}

$* p<.05$ duced movement, but is not necessary. (b) Relative displacement has not been disproved as the basis for induced movement. (c) Perceived target displacement may be produced when the background as a whole, moves relative to the stationary target, but also when only the background components are in relative motion while the relationship of the target to the boundaries of the background remains unchanged.

\section{References}

Brosgole, L. An analysis of induced motion. U. S, Naval Training Device Center, Tech. Rep. 1H-48, February, 1966a.

Brosgole, L. Change in phenomenal location and perception of motion. Percept. mot. Skills, 1966b, 23, 999-1001.

Brosgole, L. Induced autokinesis. Percept. \& Psychophys., 1967, $2,69-73$.

Carr, H. A. An introduction to space perception. New York: Longmans, Green \& Co., 1935.

Roelofs, C. O. Optische Lokalisation. Arch. Augenheilk., 1935 $109,395-415$

Sugarman, R. C. Visual localization and perception of motion as a function of eye movements. Unpublished doctoral dissertation, State University of New York at Buffalo, 1967.

Wapner, S., \& Wemer, H. Perceptual development. Worcester: Clark University Press, 1957.

\section{Notes}

1. Based, in part, on a paper presented to the Eastern Psychological Association, Boston, 1967. This work was carried out while R. S. was receiving support from NIDR Training Grant 50-780B at the State University of New York at Buffalo. Computer analysis was provided by the Computing Center at the State University of New York at Buffalo which is partially supported by NIH Grant FR-00126 and NSF Grant GP-7318.

2. Now at the Cornell Aeronautical Laboratory, Inc., Buffalo, N. Y. 